\title{
A General Bianchi Type Viscous Fluid Cosmological Model with Both Deceleration and Acceleration
}

\author{
Shri $\operatorname{Ram}^{1 *}$, S. Chandel ${ }^{1}$ and M. K. Verma ${ }^{2}$ \\ ${ }^{1}$ Department of Mathematical Sciences, Indian Institute of Technology(BHU), Varanasi -2210 05, India \\ ${ }^{2}$ Department of Mathematics, BBD NITM Lucknow-227105, India \\ Email: srmathitbhu@rediffmail.com
}

\begin{abstract}
In this paper, we obtain an anisotropic general Bianchi type cosmological model in the presence of a bulk viscous fluid. The exact solution of Einstein's field equations is obtained by utilizing a special form of time-varying deceleration parameter that gives an early decelerating and late-time accelerating model of the universe. The physical and geometrical features of the cosmological model are discussed during the evolution of the universe. We observed that the universe accelerates in time and asymptotically tends towards a static homogeneous universe.
\end{abstract}

Keywords: General bianchi model, viscosity, deceleration, acceleration.

\section{Introduction}

The evolution of spatially homogeneous and isotropic cosmological models filled with perfect fluid has been extensively studied by several cosmologists. It is certainly of interest to study cosmologies with a richer structure both geometrically and physically than the standard perfect fluid Friedmann-Robertson-Walker (FRW) models. The adequacy of isotropic cosmological models for describing the present state of the universe is no basis for expecting that they are equally suitable for describing the early stages of evolution of the universe when radiation in form of photons as well as neutrinos decoupled and the matter behaved like a viscous fluid. The observed physical phenomena such as the large entropy per baryon and the remarkable degree of the isotropy of Cosmic Microwave Background Radiation (CMBR) reveal the importance of dissipative effects in cosmology. Dissipative effects including both bulk and shear viscosities play significant roles to study early evolution of the universe. According to the grand unified theory(GUT), the phase transition and string creation are also believed to evolve viscous effects.

In recent years the introduction of viscosity in the cosmic fluid content has been found useful in explaining many important physical aspects of the dynamics of homogeneous cosmological models. Eckart [1] developed first relativistic theory of non-equilibrium thermodynamics to study the effect of viscosity. Weinberg [2] derived general form for bulk and shear viscosities and used them to evaluate the cosmological entropy prediction rate. Misner $[3,4]$ studied the effect of viscosity on the evolution of the universe and suggested that the strong dissipation, due to neutrino viscosity may considerably reduce the anisotropy of the black-body radiation.

Murphy [5] developed a uniform cosmological model filled with a fluid which possesses pressure and bulk viscosity exhibiting the interesting feature that the big-bang type singularity appears in the infinite past. $\operatorname{Gr} \phi n$ [6] investigated a viscous fluid model of the inflationary universe. Production of entropy and viscous damping of anisotropy inhomogeneous cosmological models of Bianchi type I spaces have been studied by Caderni and Fabbri [7]. Pradhan and Pandey [8], Pradhan and Singh [9], Peebles [10], Bali and Pradhan [11], Singh et al.[12], Dunn and Tupper [3], Coley and Tupper [14], Banerjee and Santos [15, 16] discussed cosmological models under the influence of both bulk and shear viscosities. Goener and Kowalewsky [17] presented a method for constructing anisotropic viscous fluid cosmological models of Bianchi type-I satisfying the barotropic equation of state. Verma and Shri Ram [18] discussed a Bianchi type III anisotropic cosmological model filled with bulk viscous fluid with time varying gravitational and cosmological constants. Shri Ram and Verma [19] obtained hypersurface-homogeneous cosmological models with time-varying gravitational and cosmological constants. Shri Ram et al. [20] investigated spatially homogeneous Bianchi type-V cosmological models with viscous fluid and heat flow in Lyra's 
geometry. Singh et al.[21] derived Bianchi type-V viscous fluid cosmological models with time varying cosmological term by assuming the rate of shear scalar and scalar expansion to be a suitable function of the average scale factors.

In this paper, we examine the possibility of a general Bianchi type cosmological model filled with bulk viscous fluid. The paper is organized as follows. The metric and field equations are given in Sect.2. Solutions of the field equations are presented in Sect.3 by assuming a special form of time-varying deceleration parameter. The geometrical and physical features of the model are discussed in Sect. 4. Some concluding remarks are given in Sect. 5 .

\section{The Metric and Field Equations}

We consider the general Bianchi type space-time in orthogonal form represented by the line-element

$$
d s^{2}=d t^{2}-A^{2} d x^{2}-B^{2} e^{-2 x} d y^{2}-c^{2} e^{-2 m x} d z^{2}
$$

where $A, B$ and $C$ are functions of cosmic time $t$ and $m$ is a constant. From Eq.(1), we can obtain special classes of Bianchi models as follows: type-III for $m=0$, type-V for $m=1$, type- $\mathrm{VI}_{0}$ for $m=-1$ and type- $\mathrm{VI}_{h}$ for all other values of $m$ where $m=h-1$ [22]. Bianchi type space-times provide spatially homogeneous and anisotropic models of the universe as compared to the homogeneous and isotropic FRW models. From these models the process of isotropization of the universe is studied through the passage of time. The simplicity of the field equations and relative ease of the solutions of Bianchi space-times are useful in constructing models of spatially homogeneous and anisotropic cosmologies.

We assume the matter content of the universe to be a bulk viscous fluid represented by the energymomentum tensor

$$
T_{i}^{j}=(\rho+\bar{p}) v_{i} v^{j}-\bar{p} \delta_{i}^{j}
$$

where $\bar{p}$ is the effective pressure given by

$$
\bar{p}=p-\xi v_{; i}^{i} .
$$

Here $\rho$ is matter energy density, $p$ the isotropic pressure, $\xi$ is the coefficient of bulk viscosity and $v^{i}$ is four velocity vector of the fluid satisfying $v^{i} v_{i}=1$. A semicolon denotes covariant differentiation. Since the bulk viscous pressure represents only a small correction to the isotropic pressure, it is reasonable to assume that the inclusion of viscous term in the energy momentum tensor does not change fundamentally the dynamics of the cosmic evolution.

For the metric (1), Einstein's field equations

$$
R_{i j}-\frac{1}{2} R g_{i j}=-T_{i j}
$$

together with Equations(2) and (3), in comoving coordinates $v^{i}=(0,0,0,1)$, lead to the following system of highly non-linear equations:

$$
\begin{gathered}
\frac{\ddot{B}}{B}+\frac{\ddot{C}}{C}+\frac{\dot{B}}{B} \frac{\dot{C}}{C}-\frac{m}{A^{2}}=-\bar{p}, \\
\frac{\ddot{A}}{A}+\frac{\ddot{C}}{C}+\frac{\dot{A}}{A} \frac{\dot{C}}{C}-\frac{m^{2}}{A^{2}}=-\bar{p}, \\
\frac{\ddot{A}}{A}+\frac{\ddot{B}}{B}+\frac{\dot{A} \dot{B}}{A B}-\frac{1}{A^{2}}=-\bar{p} \\
\frac{\dot{A}}{A} \frac{\dot{B}}{B}+\frac{\dot{A}}{A} \frac{\dot{C}}{C}+\frac{\dot{B}}{B} \frac{\dot{C}}{C}-\frac{m^{2}+m+1}{A^{2}}=\rho, \\
(m+1) \frac{\dot{A}}{A}-\frac{\dot{B}}{B}-m \frac{\dot{C}}{C}=0
\end{gathered}
$$

where an overdot denotes differentiation with respect to $t$.

For the general Bianchi model(1), we define physical parameters that are of observational importance. The spatial volume $V$ and the average scale factor $a$ are given by

$$
V=a^{3}=A B C .
$$


The expansion scalar $\theta$, shear scalar $\sigma$ and mean Hubble parameter are given by

$$
\begin{gathered}
\theta=v_{; i}^{i}=\left(\frac{\dot{A}}{A}+\frac{\dot{B}}{B}+\frac{\dot{C}}{C}\right), \\
\sigma^{2}=\frac{1}{2}\left[H_{1}^{2}+H_{2}^{2}+H_{3}^{2}\right]-\frac{1}{6} \theta^{2}
\end{gathered}
$$

where $H_{1}=\frac{\dot{A}}{A}, H_{2}=\frac{\dot{B}}{B}, H_{3}=\frac{\dot{C}}{C}$ are directional Hubble parameters.

An important observational quantity is the deceleration parameter $q$

$$
q=-\frac{a \ddot{a}}{\dot{a}^{2}}
$$

The sign of $q$ indicates whether the model inflates or not. The positive sign of $q$ corresponds to standard decelerating models whereas the negative sign indicates inflation.

Einstein's field equations are a system of highly non-linear differential equations and there is no standard method of solving them. In order to obtain physically realistic solutions, one has to make certain valid assumptions generally on the basis of physics of the problem or simply for mathematical convenience. One of them is the condition on the deceleration parameter. So far some authors proposed constant as well as time-dependent forms of $q$ and derived expressions for the average scale factor $a$, which are further used for solving the field equations. It has been observed that the cosmological models based on constant deceleration parameter by Berman [23] and linearly varying deceleration parameter proposed by Akarsu and Dereli [24] are either decelerating or accelerating. But for an universe which was decelerating in the the past and accelerating at present time, the deceleration parameter must show signature flipping (Riess et al. [25], Amendola [26]). Cunha and Lima [27] favored past deceleration and recent acceleration with high degree of statistical confidence lever by analyzing three $\mathrm{SNe}$ type $\mathrm{I}_{a}$ samples. In order to match this observation, Singh and Debnath [28] defined a special form of time-varying deceleration parameter for FRW model as

$$
q=-1+\frac{n}{a^{n}+1}
$$

where $n>0$ is a constant. Solution of equation(14) is given by

$$
H=\frac{\dot{a}}{a}=k\left(1+a^{-n}\right)
$$

where $k$ is a constant of integrating. Integrating equation(15), we obtain the average scale factor $a$ as

$$
a=\left(e^{k n t}-1\right)^{k / n} \text {. }
$$

Without loss of any generality, we can take $k=1$ and so

$$
a=\left(e^{n t}-1\right)^{1 / n}
$$

A similar form of $q$ has also been proposed by Banerjee and Das [29] in the case of Robertson-Walker space-time. Singh [30] investigated a cosmological model of Bianchi type-I with both deceleration and acceleration by using the special form $a$ in equation(17). Again using the same form of $a$, he has discussed a cosmological scenario in the case of Bianchi type-V space-time with perfect fluid source and timedependent cosmological term that describes an early deceleration and late-time acceleration [31]. Adhav et al.[32] derived early decelerating and late-time accelerating anisotropic cosmological models of Bianchi types I, III, V, $\mathrm{VI}_{0}$ and Kantowski-Sachs space-time with perfect fluid obeying the variable equation of state parameter. From equation(14), we observed that $q=n-1$ when $a=0, q=0$ for $a^{n}=n-1$ and $q<0$ for $a^{n}>n-1$. We assume that $a=0$ at $t=0$. Therefore, the universe begins with a decelerating expansion and the expansion changes from decelerating phase to an accelerating one.

Equations(5)-(8) can be expressed in terms of $q, H, \sigma^{2}$ as follows:

$$
\bar{p}=H^{2}(2 q-1)-\sigma^{2}+\frac{\left(m^{2}+m+1\right)}{3 A^{2}},
$$




$$
\rho=H^{2}-\sigma^{2}-\frac{\left(m^{2}+m+1\right)}{A^{2}} .
$$

In the next section, we obtain solutions of the field equations (5)-(9) by utilizing the special form of $a$ in equation(17) which give a class of decelerating and late time accelerating models for different values of $m$.

\section{Solutions of Field Equations}

Equation(9), on integration, yields

$$
A^{m+1}=B C^{m}
$$

where the constant of integration is taken unity. Substituting equation (20) in equation(10), we obtain

$$
V=B^{\frac{m+2}{m+1}} C^{\frac{2 m+1}{m+1}} .
$$

We assume a relation between the metric function $C$ and the spatial volume $V$ of the form $C=V^{b}$, where $b$ is a constant. Then equation(10) gives

$$
B=V^{\frac{m+1-b-2 m b}{(m+2)}} .
$$

Combining equations(20), (21) and (22), we obtain

$$
\begin{gathered}
A=V^{\frac{m+1-b+b m^{2}}{(m+1)(m+2)},} \\
B=V^{\frac{m+1-b-2 b m}{(m+2)}}, \\
C=V^{b} .
\end{gathered}
$$

In terms of the average scale factor $a$ given in equation(17), these solutions can be written explicitly in term of cosmic time $t$ as

$$
\begin{aligned}
& A=\left(e^{n t}-1\right)^{l_{1}}, \\
& B=\left(e^{n t}-1\right)^{l_{2}}, \\
& C=\left(e^{n t}-1\right)^{l_{3}}
\end{aligned}
$$

where $l_{1}=3\left(m+1-b+b m^{2}\right) / n(m+1)(m+2), l_{2}=3(m+1-b-2 m b) / n(m+2), l_{3}=3 b / n$ satisfying

$$
l_{1}+l_{2}+l_{3}=\frac{3}{n} \text {. }
$$

Hence, the metric of our solutions can be written in the form

$$
d s^{2}=d t^{2}-\left(e^{n t}-1\right)^{2 l_{1}} d x^{2}-\left(e^{n t}-1\right)^{2 l_{2}} e^{-2 x} d y^{2}-\left(e^{n t}-1\right)^{2 l_{3}} e^{-2 m x} d z^{2} .
$$

For the cosmological model equation(30), the physical and kinematical parameters have the following values :

$$
\begin{gathered}
\theta=\frac{3 e^{n t}}{\left(e^{n t}-1\right)}, \\
\sigma^{2}=\frac{\left[n^{2}\left(l_{1}^{2}+l_{2}^{2}+l_{3}^{2}\right)-3\right] e^{2 n t}}{2\left(e^{n t}-1\right)^{2}}, \\
H=\frac{e^{n t}}{\left(e^{n t}-1\right)}, \\
q=-1+n e^{-n t}
\end{gathered}
$$

From equations(18) and (19), the energy density and effective pressure are obtained as

$$
\rho=\frac{\left[5-n^{2}\left(l_{1}^{2}+l_{2}^{2}+l_{3}^{2}\right)\right] e^{2 n t}}{2\left(e^{n t}-1\right)^{2}}-\frac{\left(m^{2}+m+1\right)}{\left(e^{n t}-1\right)^{2 l_{1}}},
$$




$$
\bar{p}=\frac{4 n e^{n t}-\left[3+n^{2}\left(l_{1}^{2}+l_{2}^{2}+l_{3}^{2}\right)\right] e^{2 n t}}{2\left(e^{n t}-1\right)^{2}}+\frac{\left(m^{2}+m+1\right)}{3\left(e^{n t}-1\right)^{2 l_{1}}} .
$$

In order to determine the bulk viscosity coefficient $\xi$, we assume that the isotropic pressure and energy density satisfy the equation of state

$$
p=\gamma \rho, 0 \leq \gamma \leq 1 .
$$

Then, from equations(31)-(36), we obtain

$$
\xi=\frac{\left[3(3-\gamma)+2(2-3 \gamma) n^{2}\left(l_{1}^{2}+l_{2}^{2}+l_{3}^{2}\right)\right] e^{n t}}{18\left(e^{n t}-1\right)}-\frac{(1-3 \gamma)\left(m^{2}+m+1\right) e^{-n t}}{9\left(e^{n t}-1\right)^{2 l_{1}-1}} .
$$

\section{$4 \quad$ Physical and Geometrical Features}

We now discuss the physical and geometrical features of the cosmological model given in equation(30). We observe that the spatial volume is zero at $t=0$. At this epoch the physical parameters $\rho, p, \theta$ and $\sigma^{2}$ tend to infinity. Therefore the universe starts evolving with big-bang singularity at $t=0$. As time increases the energy density and pressure are decreasing functions of time which ultimately tend to constants as $t \rightarrow \infty$. The expansion scalar and shear scalar also tend to constants and ratio $\frac{\sigma}{\theta}$ does not tend to zero as for large time. Thus the model is anisotropic for all times.

The deceleration parameter is positive for $t<\frac{1}{n} \log n$ and is negative for $t>\frac{1}{n} \log n$. Therefore, the universe begins with decelerating expansion and the expansion in the model changes from the deceleration to acceleration at time $t=\frac{1}{n} \log n$ which indicate that the deceleration parameter has a signature flip at the epoch $t=\frac{1}{n} \log n$. The signature flip in $q$ is essential for the conclusion that the present universe is accelerating (Padmanabham and Raychoudhan [33]). Thus, this model leads to a cosmological scenario in accordance with the well known features of modern cosmology as an initial phase with decelerating expansion followed by accelerating one at late time.

From equation[17] we observe that final stage of the universe is neither accelerating or decelerating but approaches an asymptotically static homogeneous universe for specific values of the parameter which could play a vital role in the future evolution of the universe [34, 35].

The bulk viscosity coefficient is initially very large and is a decreasing function of time which ultimately tends to a constant for large time. The effect of bulk viscosity is to produce a change in perfect fluid and hence exhibits the essential influence on the character of the solution. This effect is clearly visible in the expression of isotropic pressure.

\section{Conclusion and Perspectives}

We have investigated a general Bianchi type cosmological model filled with bulk viscous fluid by assuming a time dependent form of the deceleration parameter that yields a special form of the average scale factor of the model. The model represents an expanding, shearing and non-rotating universe which starts from a big-bang singular state at $t=0$ with decelerating expansion and after a lapse of finite time the expansion in the model changes from decelerating phase to an accelerating one. The anisotropy in the model is maintained for all time. The bulk viscosity coefficient is infinite at the beginning of the model and it decreases to become a constant at late-time. We can derive the cosmological models for Bianchi types-III, $\mathrm{V}, \mathrm{VI}_{0}$ and $\mathrm{VI}_{h}$ for $m=0,1,-1$ and other values respectively where $h=m-1$.

It is worthy to mention that the assumption of the time-varying deceleration parameter in equation(14) as a function of time is ad hoc in the sense that it does not result from any known theory. However, it provides a solution which presents an appropriate description of the universe consistent with observations. The cosmological model presents a unified description of the evolution of the universe which evolves with a decelerating expansion and expands with acceleration till late time. Recent observational data (Permutter et al.[36]; Riess et al.[37, 38]; Knop et al. [39]; Tegmark et al. [40]; Spergel et al. [41]) strongly suggest this late time acceleration. Thus, the cosmological model obtained in this paper presents an appropriate description of the universe which is consistent with observations. 
Acknowledgments. The authors would like to convey their sincere thanks and gratitude to the anonymous referee for his kind suggestions for improving the paper.

\section{References}

1. C. Eckart, 'The Thermodynamics of irreversible processes III. The Relativistic theory of simple fluid.', phy. Rev., 58, pp. 919-924 (1940).

2. S. Weinberg, 'Entropy Generation and the Survival of Protogalaxies in an expanding universe', Astrophys. J., 168, pp. 175-194 (1971).

3. W. Misner, 'Transport Processes in the Primordial Fireball', Nature 214, pp. 40-41 (1967).

4. W. Misner, 'The Isotropy of the Universe', Astrophysical Journal, 151, pp. 431-457 (1968).

5. G. L. Murphy, 'Big-Bang Model Without Singularities', Phys. Rev. D, 8, pp. 4231-4233 (1973).

6. Ø. Grøn, ' Viscous inflationary universe models', Astrophys. Space Sci., 173, pp. 191-225 (1990).

7. N. Caderni, R. Fabbri, 'Production of entropy and viscous damping of anisotropy in homogeneous cosmological models', Nurvo Cimento, 44 B, pp. 228-240 (1978).

8. A. Pradhan, O. P. Pandey,'Bianchi type-I anisotropic magnetized cosmological models with varying $\Lambda$ ', Int. J. Mod. Phys. D, 12, pp.1299-1314 (2003).

9. A. Pradhan, S. K. Singh,' Bianchi type-I magnetofluid cosmological models with variable cosmological constant revisited', Int. J. Mod. Phys. D, 13, pp. 503-516 (2004).

10. P. J. E. Peebles, 'The cosmological constant and dark energy', Rev. Mod. Phys., 75, pp. 559-606, (2003).

11. R. Bali, A. Pradhan, 'Bianchi type III string cosmological models with time dependent bulk viscosity', Chin. Phys. Lett., 24, pp. 585-588(2007).

12. C. P. Singh, S. Kumar, A. Pradhan, 'Early viscous universe with variable gravitational and cosmological constants', Class. Quantum Grav., 24, pp. 455-474 (2007).

13. K. A. Dunn, B. O. J. Tupper, 'Tilting and Viscous Models in a Class of Type-VI0 Cosmologies,âAI Astrophysical Journal', Astrophys. J., 222, pp. 405-411 (1978).

14. A. A. Coley, B. O. J. Tupper, 'Viscous-Fluid Collapse', Phys. Rev. D, 29, pp. 2701-2704 (1984).

15. A. Banerjee, N. D. Santos, 'Solutions of Einstein- Yang-Mills Equations with Plane Symmetry', J. Math. Phys., 24, pp. 2635-2636 (1983).

16. A. Banerjee, N. D. Santos, 'Spatially Homogeneous Cosmological Models', Gen. Relativ. Gravit., 16, pp. 217-224 (1984).

17. F. M. Goener, F. Kowalewsky, 'Exact anisotropic viscous fluid solutions of Einstein's equations', Gen. Relativ. Gravit., 21, pp. 467-488 (1989).

18. M. K. Verma and Shri Ram, 'Bulk Viscous Bianchi Type- III Cosmological Model with Time-Dependent G and

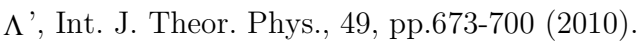

19. Shri Ram and M. K. Verma, 'Bulk viscous fluid hypersurface-homogeneous cosmological model with time varying $G$ and $\square \Lambda$ ', Astrophys. Space Sci., 330, pp. 151-156 (2010).

20. Shri Ram, M. K. Verma and M. Zeyauddin, 'Homogeneous anisotropic cosmological models with viscous fluid and heat flow in Lyra's geometry', Mod. Phys. Lett. A, 24, 1847-1856 (2009).

21. J. P. Singh, P. S. Baghel, 'Bulk viscous Bianchi type V cosmological models with decaying cosmological term', Int. J. Theor. Phys., 49, pp. 2734-2744 (2010).

22. V. R. Chirde, S. H. Shekh, P. N. Rahate, 'Two fluids viscous dark energy cosmological models with linearly varying deceleration parameter self-creation cosmology', Prespacetime Journal, 5, pp. 894-904 (2014).

23. M. S. Berman, 'Special law of variation for Hubbles parameters', Nuovo Cimento B, 74, pp.182-186 (1983).

24. Akarsu and T. Dereli, 'Cosmological models with linearly varying deceleration parameter', Int. J. Theor. Phys., 51, pp. 612-621(2012).

25. A. G. Riess, et al. , 'Support for an accelerating universe and a Glimpse of the epoch of deceleration', Astrophys. J., 560, pp. 49-71 (2001).

26. L. Amendola, 'Acceleration at Z > 1', Mon. Not. R. Astron. Soc, 342, pp. 212-226 (2003).

27. J. V. Cunha, J. S. Lima, ' Kinematic constrains to the transition red shift from SNeIa union data ', Mon. Not. R. Astron. Soc, 390, pp. $221-226$ (2008). 
28. A. K. Singh and U. Debnath, 'Accelerating Universe with a Special form of Decelerating Parameter', Int. J. Theor. Phys., 48, pp. 351-356(2009).

29. N. Banerjee and A. Das, 'Acceleration of the universe with a simple trigonometric potential', Gen. Relativ. Gravit., 37, pp.1695-1703 (2005).

30. J. P. Singh, 'A cosmological model with both deceleration and acceleration 'Astrophys. Space. Sci, 318, pp.103107 (2008).

31. J. P. Singh, 'Bianchi V cosmology with a specific Hubble parameter', Int. J. Theor. Phys., 48, pp. 2041-2049 (2009).

32. K. S. Adhav, A. S. Bansal, H. G. Ajmere, 'Early decelerating and late time accelerating anisotropic cosmological models with dynamical EoS parameter', Astrophys. Space Sci., 345, pp. 405-413 (2013).

33. T. Padmanabhan, T. Roychaudhury, 'Cosmological parameters from supernova observations: A critical comparison of three data sets ', Astron. Astrophys., 429, pp.809, (2005).

34. S. Kouwn, et al., 'Asympoticall static universe', Phys. Rev. D, 85, 063519 (2012).

35. R. A. EI-Nabulsi, 'Asymptotically static universe dominated by phantom energy', Z. Naturforsch, 70, pp. 101$108(2015)$.

36. S. Permultter, et al., 'Discovery of a supernova explosion at half the age of the universe', Nature 391, pp.51-54 (1998).

37. A. G. Riess, et al.,' Observational evidence from supernovae for an accelerating universe and a cosmological constant' Astron. J., 116, pp.1009-1038 (1998).

38. A. G. Riess, et al.,'Type-Ia supernova discoveries at $\mathrm{z}>1$ from Hubble space telescope: evidence for past deceleration and constraints and dark energy', Astrophys. J., 607, pp. 665-687 (2004).

39. A. Knop, et al. "'New Constraints on $\Omega \mathrm{M}, \Omega \Lambda$, and $\Omega \mathrm{w}$ from an Independent Set of Eleven High-Redshift Supernovae Observed with HST', Astrophys. J., 598, pp. 102-137 (2004).

40. M. Tegmark, et al., 'Cosmological parameters from SDSS and WMAP', Phys. Rev. D, 69, pp. 103501 (2004).

41. D. N. Spergel, et al., WMAP three year results: Implications for cosmology: Preprint astro-ph/ 0603449 (2006). 